\title{
REDUCIBLE HILBERT SCHEME OF SMOOTH CURVES WITH POSITIVE BRILL-NOETHER NUMBER
}

\author{
CHANGHO KEEM
}

(Communicated by Eric Friedlander)

\begin{abstract}
In this paper we demonstrate various reducible examples of the scheme $\mathscr{I}^{\prime}{ }_{d, g, r}$ of smooth curves of degee $d$ and genus $g$ in $\mathbb{P}^{r}$ with positive Brill-Noether number. An example of a reducible $\mathcal{I}^{\prime}{ }_{d, g, r}$ with positive $\rho(d, g, r)$, namely, the example $\mathcal{I}^{\prime}{ }_{2 g-8, g, g-8}$, has been known to some people and seems to have first appeared in the literature in Eisenbud and Harris, Irreducibility of some families of linear series with Brill-Noether number -1, Ann. Sci. École Norm. Sup. (4) 22 (1989), 33-53. The purpose of this paper is to add a wider class of examples to the list of such reducible examples by using general $k$-gonal curves. We also show that $\mathscr{F}^{\prime}{ }_{d, g, r}$ is irreducible for the range of $d \geq 2 g-7$ and $g-d+r \leq 0$.
\end{abstract}

\section{INTRODUCTION}

In [S] Severi has asserted with an incomplete proof that the subscheme $\mathcal{F}^{\prime}{ }_{d, g, r}$ which is the union of the irreducible components of the Hilbert scheme $\mathscr{H}_{d, g, r}$ whose general points correspond to smooth, irreducible, and nondegenerate curves of degree $d$ and genus $g$ in $\mathbb{P}^{r}$ is irreducible if $d \geq g+r$. Also in $[\mathrm{H}]$ it has been conjectured that $\mathscr{I}^{\prime}{ }_{d, g, r}$ is irreducible if the Brill-Noether number $\rho(d, g, r):=g-(r+1)(g-d+r)$ is positive.

In this paper we demonstrate various reducible examples of the subscheme $\mathscr{I}^{\prime}{ }_{d, g, r}$ with positive Brill-Noether number. Indeed an example of a reducible $\mathcal{I}_{d, g, r}^{\prime}$ with positive $\rho(d, g, r)$, namely the example $\mathscr{I}^{\prime}{ }_{2 g-8, g, g-8}$ (or other variations of it), has been known to some people (including the author), but it seems to have first appeared in the literature in [EH]. The purpose of this paper is to add a wider class of examples to the list of such reducible examples by using general $k$-gonal curves. We also show that $\mathscr{I}^{\prime}{ }_{d, g, r}$ is irreducible for the range of $d \geq 2 g-7$ and $g-d+r \leq 0$. Throughout we will be working over the field of complex numbers.

Received by the editors January 15, 1993.

1991 Mathematics Subject Classification. Primary 14C05, 14 C20.

Key words and phrases. Hilbert scheme, linear series, gonality.

Research partially supported by NSF Grant DMS 90-22140 and GARC-KOSEF. The author is grateful to MSRI, Berkeley, and MPI für Mathematik for the support and the stimulating atmosphere where a major part of this work was done. 


\section{TERMINOLOGY, NOTATION, AND SOME PRELIMINARY RESULTS}

We first recall that, given nonnegative integers $r, d$, for every point $p$ of the moduli space $\mathscr{M}_{g}$ of smooth curves of genus $g$ and any sufficiently small connected neighborhood $U$ of $p$, there are a smooth connected variety $\mathscr{M}$, a finite ramified covering

$$
h: \mathscr{M} \rightarrow U,
$$

and two varieties, proper over $\mathscr{M}$,

$$
\xi: \mathscr{C} \rightarrow \mathscr{M}, \quad \pi: \mathscr{G}_{d}^{r} \rightarrow \mathscr{M}
$$

with the following properties:

(1) $\mathscr{C}$ is a universal curve over $\mathscr{M}$, i.e., for every $p \in \mathscr{M}, \xi^{-1}(p)$ is a smooth curve of genus $g$ whose isomorphism class is $h(p)$.

(2) $\mathscr{G} r$ parametrizes pairs $(p, \mathscr{D})$, where $p \in \mathscr{M}$ and $\mathscr{D}$ is a linear system (possibly incomplete) of degree $d$ and of dimension $r$, which is denoted by $g_{d}^{r}$, on $C=\xi^{-1}(p)$.

Let $\mathscr{G}$ be the union of irreducible components of $\mathscr{G}_{d}^{r}$ whose general element corresponds to pairs $(p, \mathscr{D})$ such that $\mathscr{D}$ is a very ample linear system on $\xi^{-1}(p)=C$, i.e., $\mathscr{D}$ induces an embedding of $C$ into $\mathbb{P}^{r}$.

In order to show the irreducibility of $\mathscr{I}^{\prime}{ }_{d, g}, r$, it is sufficient to demonstrate the irreducibility of $\mathscr{G}$ since the open subset of $\mathscr{I}^{\prime}{ }_{d, g}, r$ consisting of points corresponding to smooth curves is a $\mathbb{P} G L_{r+1}$ bundle over an open subset of $\mathscr{G}$. Also we will utilize the following fact which is basic in the theory for our purposes; see $[\mathrm{ACl}]$ or $[\mathrm{H}]$ for detailed discussion and proof.

Proposition 1.1. There exists a unique component $\mathscr{G}_{0}$ of $\mathscr{G}$ which dominates $\mathscr{M}\left(\right.$ or $\left.\mathscr{M}_{g}\right)$ if the Brill-Noether number $\rho(d, g, r)$ is positive. Furthermore in this case, for any possible component $\mathscr{G}^{\prime}$ of $\mathscr{G}$ other than $\mathscr{G}_{0}$, a general element $(p, \mathscr{D})$ of $\mathscr{G}^{\prime}$ is such that $\mathscr{D}$ is a special linear system on $C=\xi^{-1}(p)$.

Remark 1.2. In the Brill-Noether range, i.e., in the range $\rho(d, g, r)>0$, we call the unique component $\mathscr{G}_{0}$ of $\mathscr{G}$ which dominates $\mathscr{M}$ the principal component. We call other possible components exceptional components.

The following facts will also turn out to be useful for our purposes; see [AC2] for the proof.

Proposition 1.3. (i) Any component of $\mathscr{G}_{d}^{r}$ has dimension at least $3 g-3+$ $\rho(d, g, r)$.

(ii) Suppose $g>0$, and let $X$ be a component of $\mathscr{G}_{d}^{2}$ whose general element $(p, \mathscr{D})$ is such that $\mathscr{D}$ is a linear system on $C=\xi^{-1}(p)$ which is not composed with an involution. Then

$$
\operatorname{dim} X=3 g-3+\rho(d, g, 2)=3 d+g-9 .
$$

(iii) The variety $\mathscr{G}_{d}^{1}$ is smooth of dimension

$$
\rho(d, g, 1)+\operatorname{dim} \mathscr{M}_{g} .
$$

By using Proposition 1.3(ii), one can prove the following fact regarding a subvariety of $\mathscr{E}_{d}^{r}$ consisting of birationally very ample linear series; see [KK]. 
Proposition 1.4. Let $\mathscr{W}$ be an irreducible closed subvariety of $\mathscr{G}_{d}^{r}, r \geq 2$, whose general element $(p, \mathscr{D})$ is such that $\mathscr{D}$ is complete, special, and birationally very ample on $C=\xi^{-1}(p)$. Then

$$
\operatorname{dim} \mathscr{W} \leq 3 d+g-4 r-1 .
$$

Corollary 1.5. Whenever

$$
2 g+1+\frac{3-3 g}{r}<d \leq 2 g-2 \quad(r \geq 3),
$$

$\mathscr{G}$ (and hence $\mathscr{I}_{d, g, r}^{\prime}$ ) is irreducible with the expected dimension $3 g-3+$ $\rho(d, g, r)$.

Proof. Suppose there exists an exceptional component $\mathscr{G}^{\prime}$ of $\mathscr{G}$. Since we are in the Brill-Noether range, by Proposition 1.1 there is an open set $\mathscr{V}$ of $\mathscr{G}^{\prime}$ whose elements consist of pairs $(p, \mathscr{D})$ such that $\mathscr{D}$ is a special very ample linear system on $C=\xi^{-1}(p)$. Consider the map

$$
\psi: \mathscr{V} \rightarrow \mathscr{G}_{d}^{\alpha}
$$

defined by $\psi(p, \mathscr{D})=(p,|D|)$, where $D \in \mathscr{D}, \alpha=\operatorname{dim}|D|$. Then by Proposition 1.4 and by noting the fact that the dimension of a fiber of $\psi$ over a point in $\psi(\mathscr{V})$ is $\operatorname{dim} \mathbb{G}(r, \alpha)$, we have $\operatorname{dim} \mathscr{G}^{\prime}=\operatorname{dim} \mathscr{V} \leq 3 d+g-4 \alpha-1+$ $(r+1)(\alpha-r)=3 d+g-1-r^{2}-r+(r-3) \alpha$.

On the other hand, by Castelnuovo theory the largest possible $\alpha$ in case $d \geq g$ is $\frac{2 d-g+1}{3}$. Thus the above inequality implies

$$
\operatorname{dim} \mathscr{G}^{\prime} \leq 3 d+g-1-r^{2}-r+(r-3) \frac{2 d-g+1}{3}<3 g-3+\rho(d, g, r),
$$

which is contradictory to Proposition 1.3(i).

Remark 1.6. (i) Corollary 1.5 was also known to Ein; see [E1]. He later gave a wider range of $d, g, r$ for which $\mathscr{I}_{d, g, r}$ is irreducible when $r \geq 5$; see [E2].

(ii) It is quite easy to show that, in case $d \geq 2 g-1, \mathscr{I}^{\prime}{ }_{d, g, r}$ is empty if $r>d-g$ and is irreducible if $r \leq d-g$; see [H, p. 61].

\section{IRREDUCIBILITY OF $\mathscr{F}^{\prime}{ }_{d, g}, r$ WITH LARGE $d$}

Theorem 2.1. $\mathscr{I}^{\prime}{ }_{d, g, r}$ is irreducible for $d \geq 2 g-7$ and $g+r \leq d, r \geq 3$.

Proof. For the case $d \geq 2 g-2$, it is a consequence of Corollary 1.5 and Remark 1.6(ii). For the case $2 g-7 \leq d \leq 2 g-3$, we proceed as follows. Let $d=$ $2 g-2-k$, where $1 \leq k \leq 5$. Suppose there exists an exceptional component $\mathscr{G}^{\prime}$ of $\mathscr{G}$. Then by Proposition 1.1, a general element $(p, \mathscr{D}) \in \mathscr{G}^{\prime}$ is such that $\mathscr{D}$ is a special linear system on $C=\xi^{-1}(p)$, i.e., $\operatorname{dim}|\mathscr{D}|>d-g$. Let $\mathscr{V}$ be an open subset of $\mathscr{G}^{\prime}$ consisting of elements $(p, \mathscr{D})$ with $\operatorname{dim}|\mathscr{D}|=\alpha>d-g$. Consider the map

$$
\Psi: \mathscr{V} \rightarrow \mathscr{G}_{k}^{k+\alpha+1-g}
$$

defined by $\Psi(p, \mathscr{D})=(p,|K-D|)$, where $D \in \mathscr{D}$ and $K$ is a canonical divisor on $C=\xi^{-1}(p)$. Then by noting the fact that the dimension of a fiber of $\Psi$ over a point in $\mathscr{G}_{k}^{k+\alpha+1-g}$ is at most $\operatorname{dim} \mathbb{G}(r, \alpha)$, we have

$$
\operatorname{dim} \mathscr{G}^{\prime}=\operatorname{dim} \mathscr{V} \leq \operatorname{dim} \mathscr{G}_{k}^{k+\alpha+1-g}+(r+1)(\alpha-r) .
$$


By Clifford's theorem and the inequality $\alpha>d-g=g-2-k$, we have $g-1-k \leq \alpha \leq g-1-\frac{k}{2}$. Thus only the following pairs for $(k, \alpha)$ 's are possible:

(i) $\{(k, g-k-1) ; 1 \leq k \leq 5\}$;

(ii) $\{(k, g-k) ; 2 \leq k \leq 5\}$;

(iii) $\{(k, g-k+1) ; k=4$ or 5$\}$.

For case (i), by inequality (2.1.1) and the hypothesis $g+r \leq d$, $\operatorname{dim} \mathscr{G}^{\prime} \leq \operatorname{dim} \mathscr{G}_{k}^{0}+(r+1)(g-k-1-r)<3 g-3+\rho(2 g-2-k, g, r)$, which is a contradiction.

For case (ii), again by (2.1.1) and the hypothesis $g+r \leq d$, we have

$$
\begin{aligned}
\operatorname{dim} \mathscr{G}^{\prime} & \leq \operatorname{dim} \mathscr{G}_{k}^{1}+(r+1)(g-k-r) \\
& =2 g-5+2 k+(r+1)(g-k-r)<3 g-3+\rho(d, g, r),
\end{aligned}
$$

which is a contradiction.

For case (iii), suppose $(k, \alpha)=(4, g-3)$. Because $\alpha=\frac{d}{2}$, any $(p, \mathscr{D}) \in \mathscr{V}$ is such that $C=\xi^{-1}(p)$ is a hyperelliptic curve by Clifford's theorem. But this is a contradiction since a hyperelliptic curve cannot have a birationally very ample special linear system.

Suppose $(k, \alpha)=(5, g-4)$. Consider $\Psi(p, \mathscr{D})=(p,|K-D|) \in \mathscr{G}_{5}^{2}$. If the complete $|K-D|$ has no base point, $|K-D|$ induces a birational map on $C=\xi^{-1}(p)$ and $g(C) \leq 6$, contrary to the hypothesis $g+r \leq d$ and $r \geq 3$. Thus $|K-D|$ has a base point, and there exists a $g_{4}^{2}$ on $C$ whence $C$ is a hyperelliptic curve by Clifford's theorem. Again this is a contradiction because there cannot exist a birationally very ample special linear system on a hyperelliptic curve.

To demonstrate the reducibility of $\mathscr{I}^{\prime}{ }_{2 g-8, g, g-8}$, we do need the following lemma whose elementary proof we omit here.

Lemma 2.2. Let $C$ be a trigonal curve of genus $g \geq 8$ with the trigonal pencil $g_{3}^{1}$. Then $\left|K-2 g_{3}^{1}\right|$ is very ample, and any $g_{6}^{2}$ is equal to $2 g_{3}^{1}$.

Theorem 2.3. (i) For $r<\frac{2 g-7}{3}, r \leq g-8$, and $r \geq 3, \mathscr{I}^{\prime}{ }_{2 g-8, g, r}$ is irreducible.

(ii) For $\frac{2 g-7}{3} \leq r \leq g-8$ and $r \geq 3, \mathscr{I}^{\prime}{ }_{2 g-8, g, r}$ is reducible with two components. Furthermore, a general element of the exceptional component is trigonal.

Proof. We use all the notation used in the proof of Theorem 2.1. Let $\mathscr{G}^{\prime}$ be an exceptional component of $\mathscr{G}$ and $\alpha=\operatorname{dim}|\mathscr{D}|$ for general $(p, \mathscr{D}) \in \mathscr{G}^{\prime}$. By Clifford's theorem, we have $\alpha=g-7, g-6$, or $g-5$.

(i) If $\alpha=g-7$ or $g-6$, one can use inequality (2.1.1) and proceed exactly as in the previous theorem to show that these cases do not occur.

(ii) If $\alpha=g-5,|K-D|=g_{6}^{2}$, where $D \in \mathscr{D}$ for a general $(p, \mathscr{D}) \in \mathscr{G}^{\prime}$. By the hypothesis $3 \leq r \leq g-8$, the map induced by $|K-D|$ on $C=$ $\xi^{-1}(p)$ is not birational. Instead, $C$ may be either hyperelliptic, trigonal, or elliptic-hyperelliptic, but $C$ cannot be hyperelliptic because a hyperelliptic curve cannot have a very ample special linear system. If $C$ is elliptic-hyperelliptic, $|K-D|=g_{6}^{2}=\phi^{*}\left(g_{3}^{2}\right)$, where $\phi$ is the map of degree 2 onto an elliptic curve $E$. Then $|\mathscr{D}|=\left|K-g_{6}^{2}\right|=g_{2 g-8}^{g-5}$ is not even birationally very ample because 
$\left|K-g_{6}^{2}-P-Q\right|=g_{2 g-10}^{g-6}$, where $P+Q=\phi^{*}(R), R \in E$. Thus $C$ cannot be elliptic-hyperelliptic.

If $C$ is a trigonal curve, $|K-D|=g_{6}^{2}=2 g_{3}^{1}$ and $|\mathscr{D}|=|D|$ is very ample by Lemma 2.2 . Thus the only possible exceptional component of $\mathscr{G}$ may arise in this way; in other words, $\mathscr{V}$ surjects onto an open set of $\mathscr{M}_{g, 3}^{1}$ if such $\mathscr{G}^{\prime}$ exists. Hence

$$
\operatorname{dim} \mathscr{G}^{\prime}=\operatorname{dim} \mathscr{V}=\operatorname{dim} \mathscr{G}_{3}^{1}+(r+1)(g-5-r) \geq 3 g-3+\rho(d, g, r),
$$

which proves the first half of the theorem.

On the other hand, suppose the above inequality holds, and let $\mathscr{W}$ be the closed subvariety of $\mathscr{G}_{d}^{r}$ whose general element $(p, \mathscr{D})$ is such that $p$ corresponds to a trigonal curve and $\mathscr{D}$ is a general $r$-dimensional subspace of $\left|K-2 g_{3}^{1}\right|$ on $C=\xi^{-1}(p)$; i.e., $\mathscr{W}$ is just the locus in $\mathscr{G}_{2 g-8}^{r}$ over trigonal curves. By the preceding discussion, $\mathscr{W}$ is indeed a component of $\mathscr{G}$ other than $\mathscr{G}_{0}$ because $\mathscr{D}$ is very ample. Furthermore, the uniqueness of such an exceptional component $\mathscr{G}^{\prime}=\mathscr{W}$ is also obvious from the preceding discussion.

\section{EXCEPTIONAL COMPONENTS OVER GENERAL $k$-GONAL CURVES}

We now construct more examples of reducible $\mathscr{F}^{\prime}{ }_{d, g, r}$ with positive BrillNoether number by using general $k$-gonal curves. We need the following lemma due to Ballico [B, Proposition 1].

Lemma 3.1. Fix positive integers $g, k, \ell$ with $k \geq 2, g \geq 2 k-2$, and $1 \leq$ $\ell \leq\left[\frac{g}{k-1}\right]$. Let $|E|=g_{k}^{1}$ be the unique pencil of degree $k$ on a general $k$-gonal curve of genus $g$. Then $\operatorname{dim}|\ell E|=\ell$.

Corollary 3.2. Fix positive integers $g, k, \ell$ with $k \geq 3, g \geq 2 k-2$, and $1 \leq \ell \leq\left[\frac{g}{k-1}\right]-2$. Let $|E|=g_{k}^{1}$ be the unique pencil of degree $k$ on a general $k$-gonal curve $C$ of genus $g$. Then for any $P, Q \in C, \operatorname{dim}|\ell E+P+Q|=\ell$. Proof. We first claim that $\operatorname{dim}|\ell E+P|=\ell$ for any $P \in C$. Suppose $\operatorname{dim}|\ell E+P|=\ell+1$ for some $P \in C$. By Lemma 3.1, $\operatorname{dim}|(\ell+1) E|=$ $\operatorname{dim}\left|\ell E+P+E^{\prime}-P\right|=\operatorname{dim}|\ell E+P|=\ell+1, E^{\prime} \in|E|$. Then $E^{\prime}-P \succ 0$ is the base locus of $|(\ell+1) E|$, which is in fact base-point-free.

Suppose that $\operatorname{dim}|\ell E+P+Q|=\ell+1$ for some $P, Q \in C$. By the first claim and Lemma 3.1, we have $\ell+1=\operatorname{dim}|\ell E+P+Q|=\operatorname{dim}|(\ell+1) E|=$ $\operatorname{dim}|(\ell+1) E+P|=\operatorname{dim}\left|\ell E+P+Q+E^{\prime \prime}-Q\right|$, where $E^{\prime \prime} \in|E|$ and $E^{\prime \prime}-Q \succ 0$. Then $E^{\prime \prime}-Q$ is the base locus of the linear system $|(\ell+1) E+P|$, but this is a contradiction because the actual base locus of $|(\ell+1) E+P|$ is $P$.

Lemma 3.2 implies the following immediate corollary.

Corollary 3.3. Let $g, k, \ell$ be positive integers such that $k \geq 3, g \geq 2 k-2$, and $1 \leq \ell \leq\left[\frac{g}{k-1}\right]-2$. Let $C$ be a general $k$-gonal curve with the unique pencil $|E|$ of degree $k$. Then $|K-\ell E|$ is very ample.

Theorem 3.4. Let $g, k, \ell, r$ be integers such that $k \geq 3, r \geq 3,2 \leq \ell \leq$ $\left[\frac{g}{k-1}\right]-2, \frac{2 g+2-2 k}{\ell+1}-1<r \leq g-2-\ell k$, and $d=2 g-2-\ell k$. Then $\mathcal{F}_{d, g, r}^{\prime}$ is reducible with at least one exceptional component containing the family of general k-gonal curves. 
Proof. By Corollary 3.3, there exists a family $\mathscr{A}$ of $k$-gonal curves in $\mathbb{P}^{r}$ of degree $2 g-2-\ell k$ embedded by a general $r$-dimensional subsystem of $\left|K-\ell g_{k}^{1}\right|$. Furthermore

$$
\begin{aligned}
\operatorname{dim} \mathscr{A} & \geq \operatorname{dim} \mathscr{M}_{g, k}^{1}+\operatorname{dim} \mathbb{G}(r, g-\ell k+\ell-1)+\operatorname{dim}\left(\text { Aut } \mathbb{P}^{r}\right) \\
& >3 g-3+\rho(d, g, r)+(r+1)^{2}-1
\end{aligned}
$$

in the given range of $g, k, \ell$, and $r$. Thus there must be an exceptional component containing the family of general $k$-gonal curves, and hence $\mathscr{I}^{\prime}{ }_{d, g, r}$ is reducible.

Remark 3.5. (i) In all the examples we demonstrated so far, we deliberately chose the numbers $d, g$, and $r$ so that the Brill-Noether number was positive, in particular, $\rho(d, g, r) \geq g$. On the other hand, one can come up with many examples, e.g., $\mathscr{I}^{\prime}{ }_{2 g-2-\ell k, g, g-\ell k+\ell-1}$ which violate the so-called Brill-NoetherPetri Principle (see [EH, §2]) for those $g, k$, and $\ell$ in the same range as in Theorem 3.4, and in these cases the Brill-Noether number becomes negative.

(ii) If $\ell=2$ in Theorem 3.4, one can show that the family of general $k$ gonal curves contained in an exceptional component of $\mathscr{I}^{\prime}{ }_{2 g-2-2 k, g, r}$ is indeed dense in the component.

\section{REFERENCES}

[AC1] E. Arbarello and M. Cornalba, $A$ few remarks about the variety of irreducible plane curves of given degree and genus, Ann. Sci. École Norm. Sup. (4) 16 (1983), 467-483.

[AC2] __ Su una congettura di Petri, Comment. Math. Helv. 56 (1981), 1-38.

[B] E. Ballico, A remark on linear series on general k-gonal curves, Boll. Un. Mat. Ital. A (7) 3 (1989), 195-197.

[E1] L. Ein, Hilbert scheme of smooth space curves, Ann. Sci. École Norm. Sup. (4) 19 (1986), 469-478.

[E2] - Irreducibility of the Hilbert scheme of smooth space curves, Proc. Sympos. Pure Math., vol. 46, Amer. Math. Soc., Providence, RI, 1987, pp. 83-86.

[EH] D. Eisenbud and J. Harris, Irreducibility of some families of linear series with Brill-Noether number -1, Ann. Sci. Ėcole Norm. Sup. (4) 22 (1989), 33-53.

[H] J. Harris, Curves in projective space, Presses Univ. Montréal, Montréal, 1982.

[KK] C. Keem and S. Kim, On the Clifford index of a general $(e+2)$-gonal curve, Manuscripta Math. 63 (1989), 83-88.

[S] F. Severi, Vorlesungen über algebraische Geometrie, Teubner, Leipzig, 1921.

Department of Mathematics, Seoul National University, Seoul, 151-742 Korea

Current address: Max-Planck-Institut für Mathematik, Gottfried-Claren-Strasse 26, D-5300

Bonn 3, Germany

E-mail address: CKEEM@KRSNUCC1. BITNET

CKEEMOMPIM-BONN. .MPG.DE 